\title{
UAV-Borne photogrammetry: a low cost 3D surveying methodology for cartographic update
}

\author{
Gabriella Caroti $^{1, *}$, Andrea Piemonte $^{1}$, and Riccado Nespoli ${ }^{2}$ \\ ${ }^{1}$ Civil and Industrial EngineeringDepartment (DICI), University of Pisa, Largo Lucio Lazzarino 1, \\ Pisa, Italy \\ 23D A.P.S. (Aero PhotogrammetricSurveys), Via F.Redi 78/a3, Arezzo, Italy
}

\begin{abstract}
Territorial management requires the most possible up-to-date mapping support of the status quo. Regional scale cartography update cycle is in the medium term (10 to 15 years): therefore, in the intervening time between updates relevant Authorities must provide timely updates for new works or territorial changes. Required surveys can exploit several technologies: ground-based GPS, Terrestrial Laser Scanning (TLS), traditional topography, or, in the case of wider areas, airborne photogrammetry or laser scanning. In recent years UAV-based photogrammetry is becoming increasingly widespread as a versatile, lowcost surveying system for small to medium areas. This surveying methodology was used to generate, in order, a dense point cloud, a high resolution Digital Surface Model (DSM) and an orthophotograph of a newly built marina by the mouth of the Arno river in Pisa, Italy, which is not yet included in cartography. Surveying activities took place while the construction site was in operation. Case study issues surfaced in the course of the survey are presented and discussed, suggesting 'good practice' rules which, if followed in the survey planning step, can lessen unwanted effects due to criticalities. Besides, results of quality analysis of orthophotographs generated by UAV-borne images are also presented. Such results are discussed in view of a possible use of orthophotographs in updating medium- to large-scale cartography and checked against existing blueprints.
\end{abstract}

\section{Introduction}

Technological evolution referred to methods and methodologies for furthering knowledge of land and its components has gained an ever increasing importance in the evolution of many applied sciences. New technologies and methodologies in surveying have led, in the last decade, to major innovations in Geomatics. Such innovations have disclosed new boundaries and solutions for the multidisciplinary integrated approach to collect 2- and/or 3-D metrical data and related information for informative theming [1]. Digital surveying represents one of the main innovations brought along by evolution in surveying

${ }^{*}$ Correspondingauthor: gabriella.caroti@unipi.it 
methodology. Terrestrial and Airborne Laser Scanning and the image-based modeling techniques yield dense 3-D point clouds of both natural and built areas by means of simplified procedures, so that potential users are numerous and with vast cultural differences. Point clouds derived from each of these methodologies differ deeply as to definition and properties, which on turn define the best application field: point clouds derived from laser scanning are best suited to generation of high-definition geometrical models, whereas digital photogrammetry is best suited for 3D models with high-definition textures [2]. This approach has been tested on different occasions on landscapes [3-6] as well on architecture and cultural heritage [7-9] and infrastructures [10,11].

Lately, Unmanned Aerial Vehicles (UAVs) have been introduced in Geomatics as data collection systems for low altitude photogrammetric surveying [12]. High-resolution imagery, acquired using UAVs and processed by structure from Motion (SfM) and MultiView Stereo (MVS) photogrammetry produce 3D point-clouds whose accuracy ranges from sub-meter level to a few centimeters [13-15]. New researches in this field introduce this methodology as a promising technology, providing new opportunities also for map updating $[16,17]$.

Goals for this work include:

1. Check of the usability of the UAV-based survey for updating of medium- to largescale cartography and of the match with dockyard drafts;

2. Emphasize critical issues of UAV-based surveys in dockyard areas close to bodies of water.

\section{Materials and methods}

\subsection{Test area}

The case study under investigation refers to the area where the touristic port of the Marina di Pisa resort currently stands.

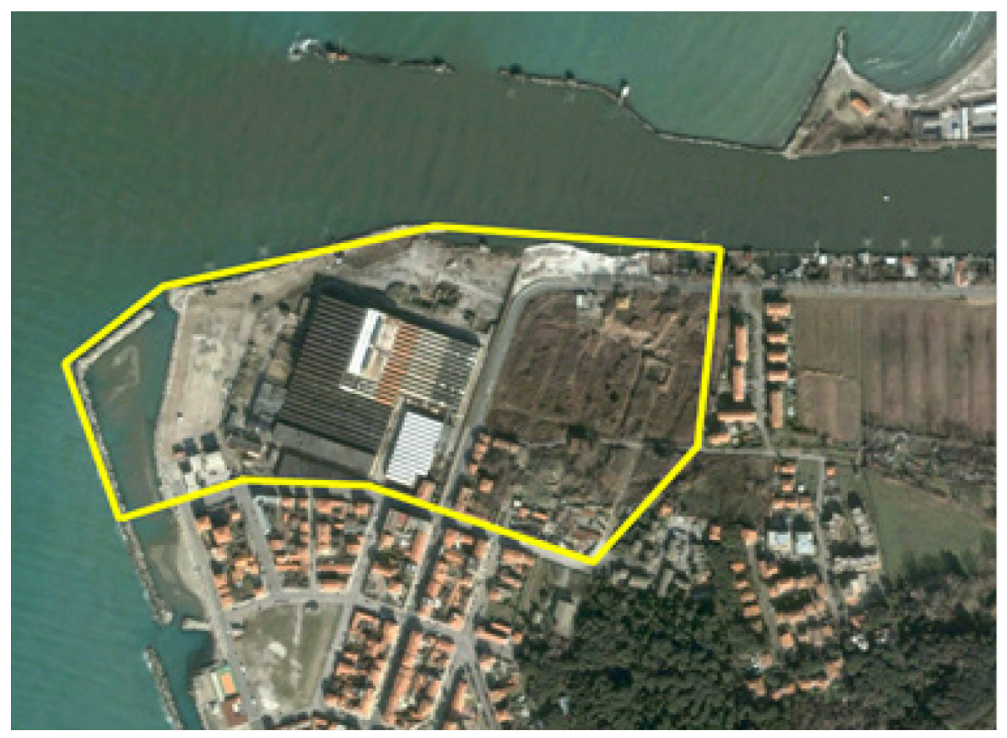

Fig. 1. Area intended for marina construction, before work - October 2007 (image from Google Earth) 
The marina is located on the Pisa seaside at the mouth of the Arno River, and is included in both the urban fabric and the Regional Park of Migliarino, San Rossore and Massaciuccoli. The area where the marina currently rises was home to a FIAT industrial plant for car manufacturing. Following the cessation of activities in 1988, the decommissioned plant was left in a state of ever worsening decay (Figure 1). At the end of the 2000s, a recovery and rehabilitation plan was approved for the area, providing its reclamation and securing, as well as its new intended use as marina with related commercial and residential spaces.

This work has dramatically altered the appearance of the mouth of the Arno River and the urban layout of Marina di Pisa (Figure 2), and medium- to large-scale cartography of the area is no longer representative of its actual conditions (Figure 3).

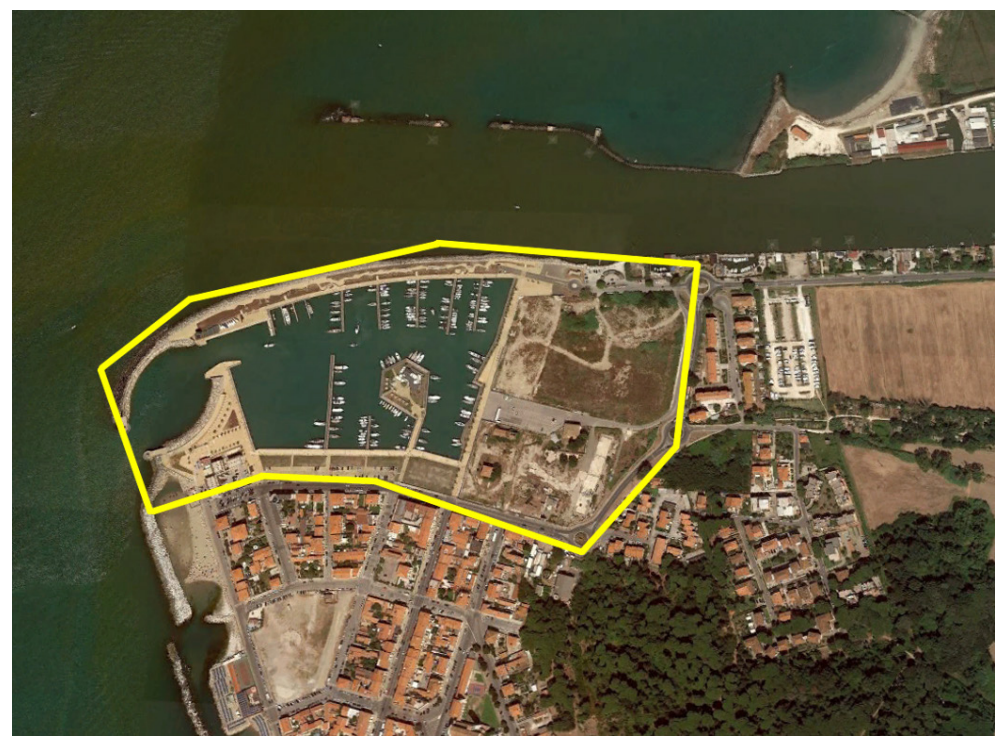

Fig. 2. Marina area in 2016 (image from Google Earth)

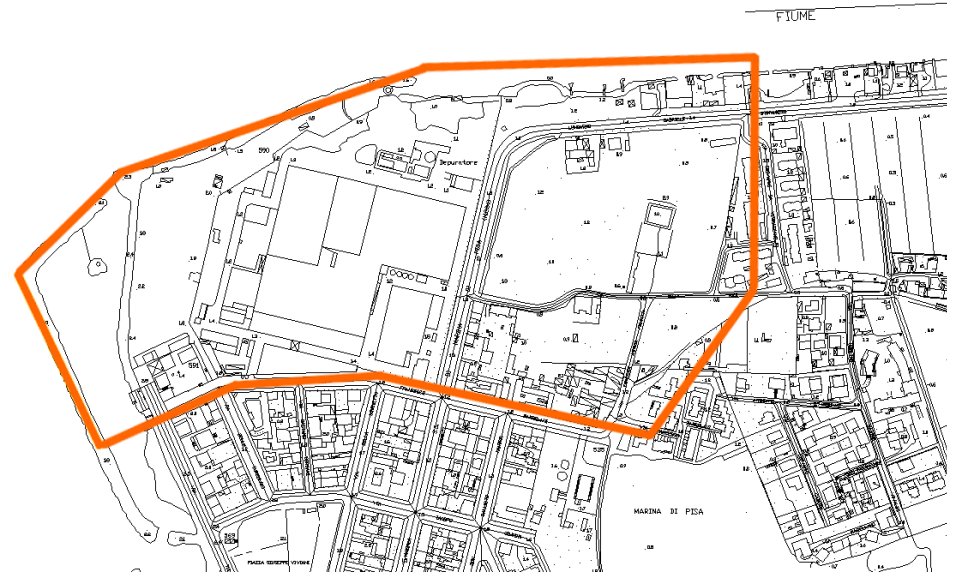

Fig. 3. 1:2000 regional cartogaphy abstract highlighting the industrial plant area where the marina has been built 
The present investigation, framed in the foresaid context, aims at assessing the potential, in terms of detail and accuracy, of UAV-based photogrammetry for timely updates of new works and landscape modifications during the period intervening between updates of medium- to large-scale cartography.

In order to assess product quality, Ground Control Points (GPs) have been compared against project documents provided by the dockyard site management; besides, the UAVbased survey has been compared against Google Earth.

\subsection{Survey methodologies}

Actual survey operations have been carried out when the construction site was still operating, following a site inspection in order to check the status quo of the area and dockyard organization. Flight area coverage and times, as well as take-off and landing sites, have been defined in collaboration with works direction to ensure maximum safety. The flight has taken place during the lunch break, covering the north pier area, as some vessels were already moored in other zones of the marina (Figure 4).

The flight plan was designed for a restitution scale greater than 1:1000, providing for a coverage of approximately 9 hectares with a total flight length of about $1200 \mathrm{~m}$

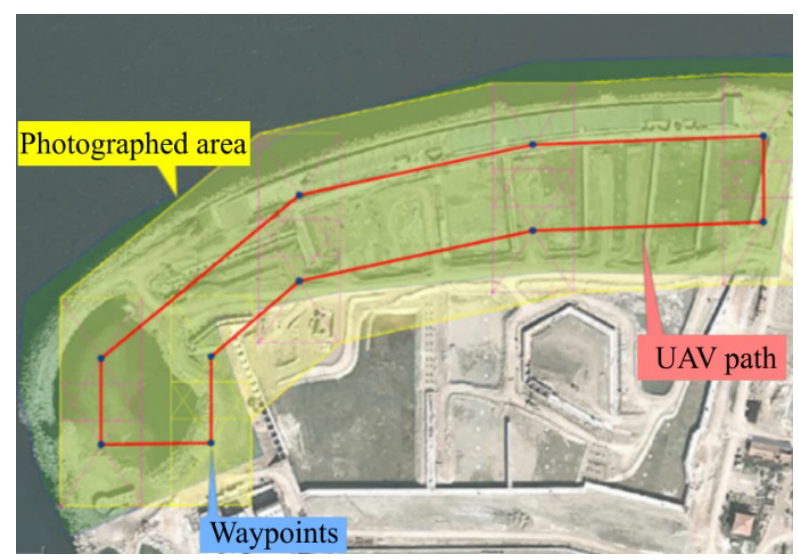

Fig. 4. Covered area.

The airborne photogrammetry survey has been carried out by means of an OnyxStarhexacopter (Figure 5) fitted with a Sony NEX 7 digital camera, whose technical features are listed below:

1. Weight: $300 \mathrm{~g}$

2. Sensor dimensions: $23.5 \mathrm{~mm} \times 15.6 \mathrm{~mm}$

3. Sensor definition: 6000pixel x 4000 pixel

4. Optics: $18-55 \mathrm{~mm}$ zoom, set in Wide mode ( $\mathrm{f} \cong 19 \mathrm{~mm}$ )

Planning of image collection provided for pseudo-nadiral shots, with horizontal strips; flight height was $\cong 80 \mathrm{~m}$, with Ground Sampling Distance (GSD) $\cong 2 \mathrm{~cm} / \mathrm{px}, 85 \%$ overlap along both axes, and theoretical coverage of about $100 \mathrm{~m} \times 65 \mathrm{~m}$. 


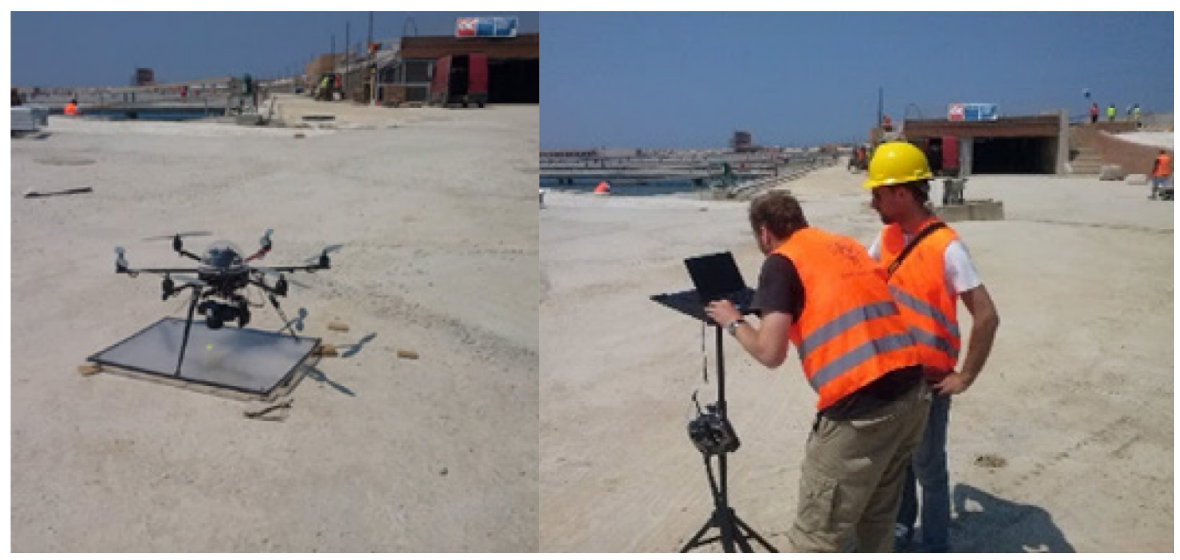

Fig. 5. Flight preparation.

Photogrammetric processing of the collected images was carried out by means of Agisoft'sPhotoScan commercial software. PhotoScan implements SfM and MVS photogrammetric algorithms, and its potential in generating 3D models from point clouds are well documented in literature [18].

In this case, the software has been used to generate a Dense Digital Surface Model (DDSM) and an orthophotograph of the object area. Control Points (CPs) have been uniformly spread across the area and signalized; their coordinates have been surveyed by RTK GPS.These points have been used for the definition of external and internal orientation parameteres of the images, by means of a self-calibration procedure built into the bundle block adjustment, in order to scale and georeference the photogrammetric survey.

In the processing step, some of these points were used solely for accuracy checks as CPs, while the remaining were used as GCPs to compute the photogrammetric model [1921].

\section{Results and discussion}

The present case study has highlighted some application peculiarities of potential interest for implementation of the survey methodology in construction and coastal sites.

Airborne image collection has pointed out some critical issues in the survey area which were not detected in previous inspections. Firstly, large construction sites are complex contexts, where works of different kind pursue time and cost optimization, which on turn leads to adapt local situations and logistics on an almost daily basis. In the present case, this required on site modification of the spot chosen for takeoff and landing, as well as of the flight plan. The spot chosen for takeoff and landing was a yard on the edge of the site, with dusty, sandy ground, which entailed a harmful event in the first flight. In fact, the turbulence due to the UAV rotors lifted the dust, impeding correct operation of two components of the surveying system, i.e. the proximity sensor (range meter) and the camera infrared sensor. As for the former, a ground proximity signal continuously fed to the autopilot system caused the UAV to rise uncontrollably, until manual flight mode was switched on, allowing for landing. On the other hand, the infrared sensor malfunction caused a stop in automatic image shooting, since the procedure was controlled by the sensor itself. The problem was solved by using a raised stand as takeoff platform. 

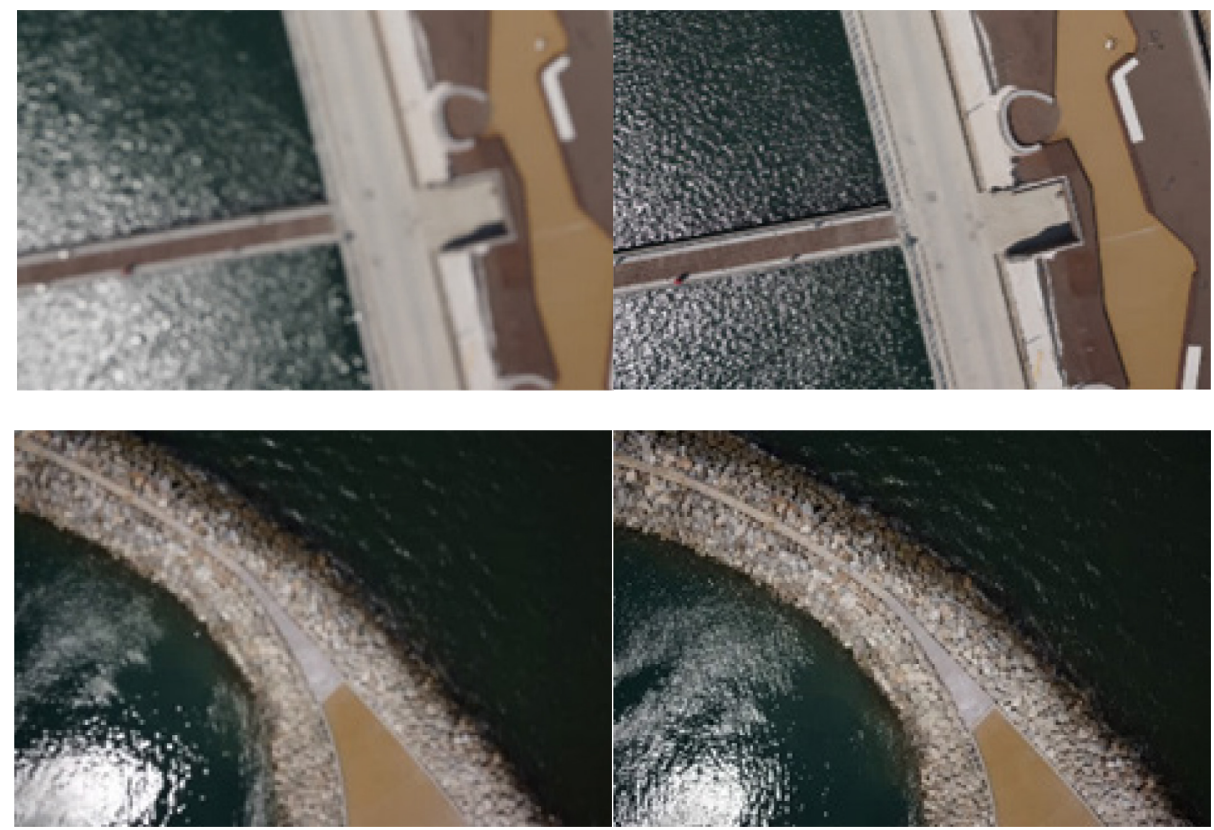

Fig. 6. Focusing issues on water bodies

Another early problem referred to use automatic focus mechanisms on mostly submersed areas (Figure 6). Water surface is in fact too homogeneous for automatic focusing to work correctly, which caused blurred images in some cases and image skipping in others. This problem has been solved by setting manual focus at infinity, as the flight level was greater than the hyperfocal distance.

A further issue, providing useful directions for flight planning, is wind assessment, even more so in this case. In fact, the marina area often features breezes, which can on occasion grow stronger; these, in turn, have even greater influence on lighter aircrafts, such as UAVs. An early test showed that strong crosswinds caused higher battery consumption, due to corrections applied by autopilot to keep the aircraft in route, reducing flight range and coverable area. Planning strips so to avoid crosswind optimizes range and flight plan compliance. The final flight focused on the north piers and large vessels mooring dock.

Image orientation errors amounted to about .5 pixels. Subsequent input of GCPs and CPs in the processing allowed for quality assessment of the DDSM obtained by photogrammetric processing. Maximum error values in the $10 \mathrm{~cm}$ range are compliant with the research goals and in accordance with literature on the subject.

Given good quality as regards flight planning, image collection and GCPs survey, expected planimetric errors are one to three times pixel size on the object, with elevation error roughly doubling the former. The orthophotograph of the test area has been generated by the PhotoScan software (Figure 7) and georeferenced in the Technical Regional Cartography system. 


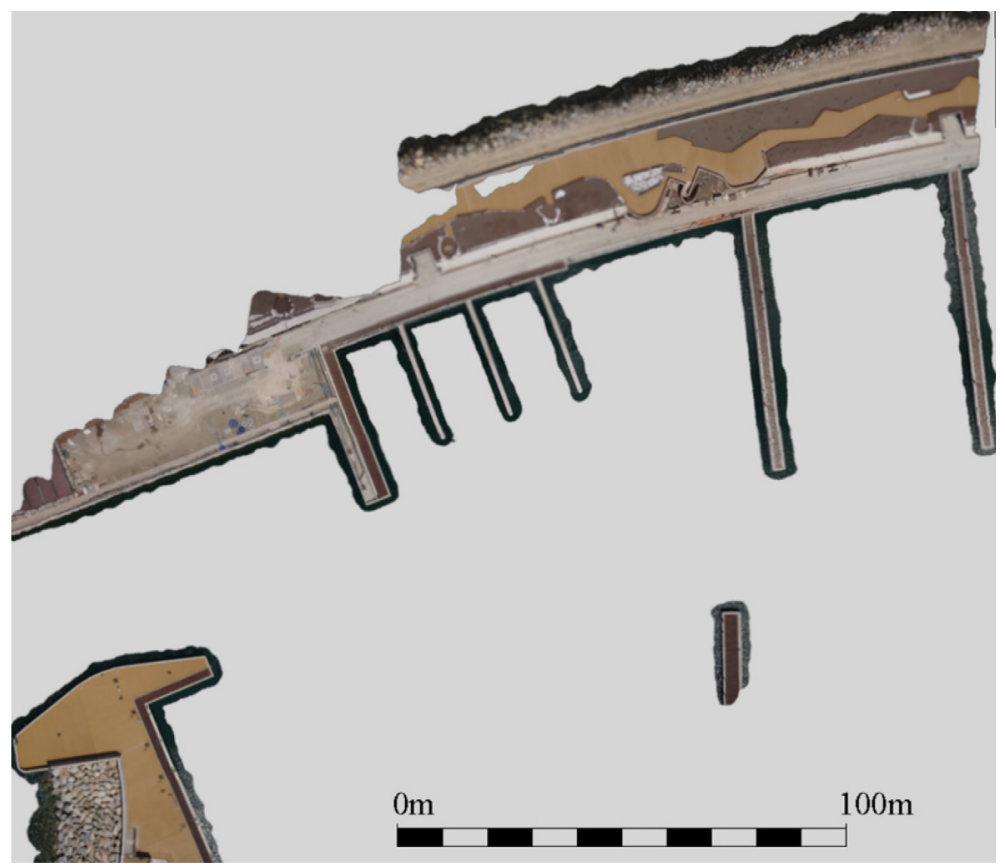

Fig. 7. Orthophotograph of the North-West part of the surveyed area (Figure 2)

A further comparison has been made against the marina blueprint, also georefenced in the Technical Regional Cartography system. This displayed an excellent compliance within the foresaid limitations (Figure 8). The area of the shorter piers showed a planimetric deviation amounting to about $80 \mathrm{~cm}$, which could suggest a systematic error (Figure 9). On the other hand, planimetric positioning of other structures posed no similar issues, as shown for the longer piers located in close proximity (Figure 8 right).

In order to detect the origin of this anomaly, a RTK-GPS survey has been carried out on 10 points, homogeneously spread across the test area and detectable in both documents. Its results showed that the actual position of the short piers differs from their planned position, so that any deviation detected by the comparison is not due to survey methodology

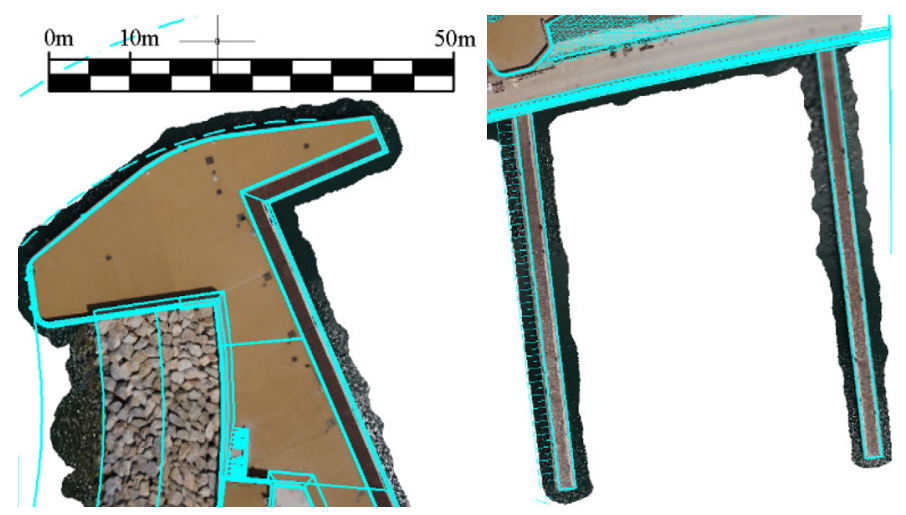

Fig. 8. Comparison areas with $10 \mathrm{~cm}$ range compliance. On the left the pier entrance in the West part of the surveyed area (Figuera 2$)$. On the right the long $(\sim 60 \mathrm{~m})$ piers in the North part of the surveyed area (Figure 2). 


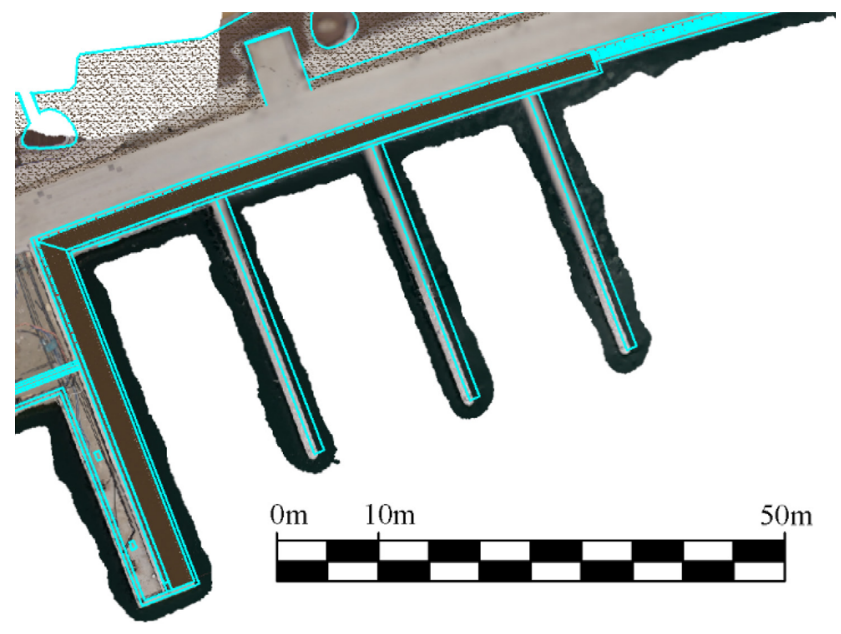

Fig. 9. Short ( $\sim 30 \mathrm{~m})$ piers: deviations $\cong 80 \mathrm{~cm}$. North-West of the surveyed area (Figure 2$)$.

The discordance between blueprint and actual position is corroborated by overlaying the orthophotograph with the corresponding Google Earth image (Figures 10 and 11).

In order to register the model orthographic projection in the same reference system of Google Earth service, it was exported by SfM software in "kmz" format.

It is of course advisable to keep in mind that accuracy Google Earth renderings is in the 1:10.000 range, anyway a first qualitative analysis denotes good compliance. Availability of up-to-date images in Google Earth can provide a tool for updating medium-scale cartography. When instead higher detail levels are required, tools as UAV-based photogrammetry may allow updates of small cartography portions with comparatively low costs. The UAV-based survey has been checked for accuracy by means of the CPs, while images shown below highlight the greater resolution of images obtained by low-altitude surveys.

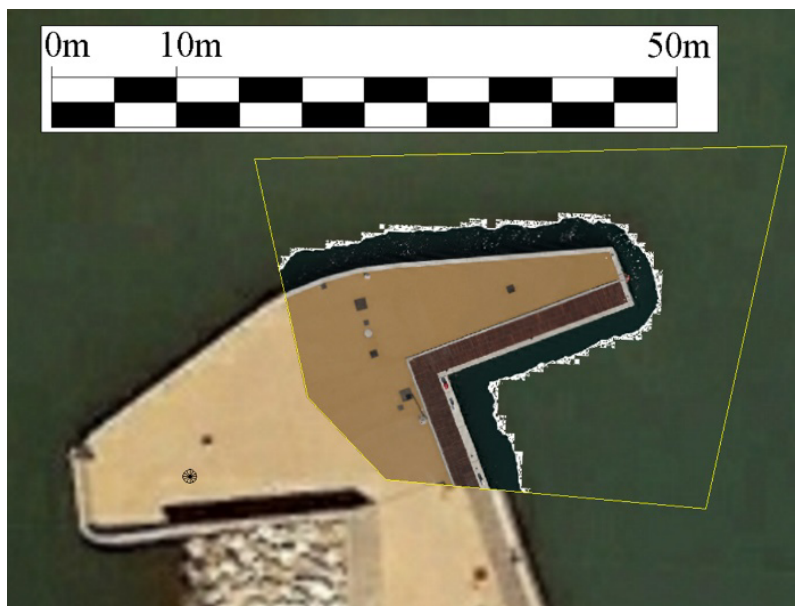

Fig. 10. Google Earth's map and orthophoto overlay. West part of the surveyed area. 


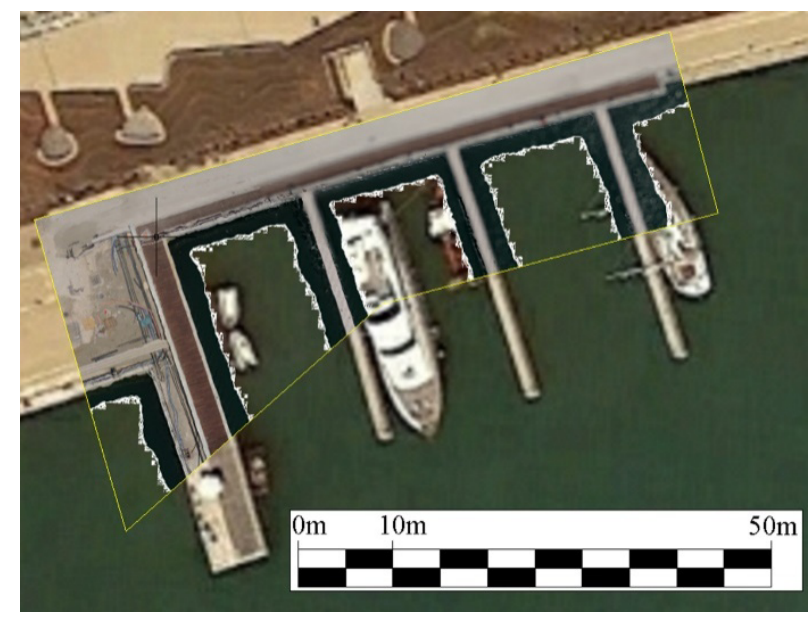

Fig. 11. Google Earth's map and orthophoto overlay. North part of the surveyed area.

\section{Conclusion}

UAV-based surveys in seaside construction sites face peculiar operating issues. The present investigation has shown that application of the methodology with proper precautions allows for medium- to large-scale map updates in the intervening time required for official updates. The different checks, carried out on the GCPs, on dockyard blueprints and on Google Earth maps, have shown that orthophotographs produced from airborne photogrammetry meet accuracy and detail requirements for cartography, aside from providing high radiometric resolution which facilitate reading of landscape changes due to new works.

Thanks are due to ASTRO (ApplicazioniScientifiche e Topografiche per ilRilievoOperativo - Scientific topogaphic applications for field surveying) laboratory technicians Dr. Andrea Bedini and Dr. Jessica Micheloni, to Ph.D. candidate Dr. Isabel Martínez-Espejo Zaragoza and to Pisa Marina dockyard management.

\section{References}

1. E.M. Cepolina, G. Caroti, A. Piemonte, P.G. Rojas,17th Int Conference on Harbor, Maritime and Multimodal Logistics Modelling and Simulation, 51 (2015).

2. F. Chiabrando, V. Di Pietra, A. Lingua, P.Maschio, F.Noardo, G.Sammartano, and A. Spanò,Int Arch Photogramm, Remote Sensing and Spatial Information Sciences,XLIB5, 413 (2016)

3. J. Roelensa, S. Dondeynea, J. Van Orshovenb, J. Dielsa,Int JApplEarth Obs, 53, 64 (2016)

4. G. Caroti, A. Piemonte, M. Redini, ApplGeomatics, 7 (4), 243 (2015)

5. G. Caroti, A. Piemonte, M.Redini, Int Arch Photogramm, Remote Sensing and Spatial Information Sciences,XL-5/W3, 99 (2013)

6. G. Caroti, F.Camiciottoli, A. Piemonte, M.Redini,Int Arch Photogramm, Remote Sensing and Spatial Information Sciences,XL-5/W3, 51 (2013)

7. P.G. Robleda, G. Caroti, I.Martínez-Espejo Zaragoza, A.Piemonte,Int Arch Photogramm, Remote Sensing and Spatial Information Sciences,XLI-B5, 391 (2016) 
8. M.G. Bevilacqua, G. Caroti, I. Martínez-Espejo Zaragoza, A. Piemonte,Remote Sensing, 8 (3), 1 (2016)

9. G. Caroti, I. Martínez-Espejo Zaragoza, A. Piemonte, Int Arch Photogramm, Remote Sensing and Spatial Information Sciences,XL-5/W4, 285 (2015)

10. M. Castro, S. Lopez-Cuervo, M.Paréns-González, C. de Santos-Berbel,Surv Rev, 48 (350), 309 (2016)

11. G. Caroti, A. Piemonte, SurvRev, 42 (315), 92 (2010)

12. I. Colomina, P. Molina, ISPRS J Photogramm Remote Sensing, 92, 79 (2014)

13. M.Gerke,H. J. Przybilla,PhotogrammFernerkun, 1, 17 (2016)

14. S.Harwin, A.Lucieer, Remote Sensing, 4, 1573 (2012)

15. F. Nex,F. Remondino, Applied Geomatics, 6 (1), 1 (2014)

16. S. Crommelinck, R. Bennett, M.Gerke, F.Nex, M.Y. Yang, G.Vosselman, Remote Sensing, 8 (8), 1 (2016)

17. M. Cramer, S. Bovet, M.Gültlinger, E.Honkavaara, A. McGill, M.Rijsdijk, M. Tabor, V.Tournadre, Int Arch Photogramm, Remote Sensing and Spatial Information Sciences,XL-1/W2, 93 (2013)

18. F. Remondino, M.G. Spera, E. Nocerino, F. Menna, F.Nex,PhotogrammeRec, 29(146), 144 (2014)

19. K. Thoeni, D.E.Guccione, M.Santise, A. Giacomini, R.Roncella, G. Forlani, IntArchPhotogramm, Remote Sensing and Spatial Information Sciences,XLI-B5, 909 (2016)

20. M. Jarzabek- Rychard, M.Karpina, Int Arch Photogramm, Remote Sensing and Spatial Information Sciences, XLI-B1, 1121 (2016)

21. G. Caroti, I.Martínez-Espejo Zaragoza, A.Piemonte, Int Arch Photogramm, Remote Sensing and Spatial Information Sciences, XL-1/W4, 103 (2015) 